\title{
Diseño y Validación de un Instrumento de Evaluación de Competencias para la Gestión de la Carrera Emprendedora
}

\author{
Design and Validation of a Skills Assessment Instrument for Managing \\ Entrepreneurial Career
}

\author{
María Fe Sánchez-García ${ }^{1}$ y Magdalena Suárez-Ortega²
}

\begin{abstract}
Resumen
Este trabajo tiene como finalidad diseñar un instrumento para evaluar las competencias que están presentes en la carrera emprendedora y que permiten gestionar los talentos conducentes al éxito en el mundo empresarial. Incluye dos escalas que evalúan respectivamente las competencias emprendedoras y las competencias generales de gestión de la carrera. Se realiza un estudio de validez de contenido mediante el juicio de 16 expertos, aplicándose a una muestra de empresarios consolidados y emprendedores en transición $(n=248)$. Se estudia su estructura factorial (mediante análisis factoriales exploratorio y confirmatorio) confirmándose la validez del modelo obtenido y comprobando su consistencia interna ( $\alpha=.868$ y .905). El análisis de conglomerados identifica tres perfiles relevantes. Se concluye acerca de la idoneidad y utilidad del instrumento, y se discute sobre la caracterización e implicaciones de los perfiles competenciales hallados con relación a las variables sociodemográficas.
\end{abstract}

Palabras clave: emprendedores, carrera emprendedora, competencias, gestión de la carrera, evaluación

\begin{abstract}
The purpose of this work is to design an instrument to assess the competencies that exist in the entrepreneurial career, which allow to manage the conducive abilities in order to be successful in the business environment. It includes two scales that evaluate respectively the entrepreneurial competences and the management general skills of the career. A validity study of content has been made with the trial of 16 experts, applying it to a sample of employers and entrepreneurs in transition $(n=248)$. The factorial structure has been analysed (through exploratory and confirmatory factorial analysis), confirming the validity of the model obtained and checking its internal consistency $(\alpha=.868$ and .905). The cluster analysis identifies three relevant profiles. The study concludes about the appropriateness and usefulness of the instrument and discusses the characterization and implications of the competence profiles found with regard to the sociodemographic variables.
\end{abstract}

Keywords: entrepreneurs, entrepreneurial career, skills, career management, assessment

\footnotetext{
${ }^{1}$ Doctora en Ciencias de la Educación. Universidad Nacional de Educación a Distancia. Profesora Titular. Juan del Rosal, 14, 28040 Madrid, España. Tel.: +34 913988275. Correo: mfsanchez@edu.uned.es

${ }^{2}$ Doctora en Ciencias de la Educación. Universidad de Sevilla. Profesora Titular. Pirotecnia, s/n, 41013 Sevilla, España.

Tel.: +34 955420632. Correo: msuarez@us.es
} 


\section{Introducción}

El neoliberalismo económico y los fenómenos asociados a nuevas formas deprecariedad laboral, unidos a ciertas políticas públicas de empleo, empujan a muchas personas a modificar sus esquemas sobre el trabajo entendido de forma tradicional y asalariada, para empezar a contemplarel autoempleo como una alternativa real (Valencia Agudelo, 2012). Surge así un colectivo creciente de emprendedores que se une al universo de los autónomos y pequeños empresarios que ya han alcanzado un grado de consolidación de su carrera emprendedora.

El emprendimiento consiste en descubrir, evaluar y explorar una oportunidad de negocio para ofrecer a terceros un producto o servicio (Shane \& Venkataraman, 2000). El proceso de lanzamiento a la carrera emprendedora es una transición importante en la trayectoria vitalprofesional, que frecuentemente se ha visto precedida de un momento crítico ya sea de reflexión personal, de crisis sobre la propia identidad profesional, o de replanteamiento existencial (Schlossberg, 2005; Sørensen \& Sharkey, 2014).

De acuerdo con los datos del informe GEM (Global Enterpreneurship Monitor 2014), en España el mayor porcentaje de emprendedores lo hicieron por oportunidad, siendo una característica del emprendimiento su heterogeneidad en relación con variables sociodemográficas tales como el nivel educativo, la experiencia laboral previa o la edad (Gómez-Araujo, Lafuente, Vaillant, \& Gómez Núñez, 2015).

Desde el ámbito de la psicología social, numerosos estudios analizan el proceder emprendedor apoyándose en la teoría del comportamiento planificado (Theory of Planned Beahaviour, TPB) de Ajzen (1991). Esta teoría destaca la importancia de la actitud y del control percibido, como los factores que determinan el comportamiento intencional, el cual es producto de tres tipos de creencias: (a) las referidas a las consecuencias probables del comportamiento, (b) las vinculadas a las expectativas normativas de otros (presión social percibida), y (c) aquellas asociadas a lo que puede facilitar o impedir el desempeño del comportamiento. También la teoría de Shapero sobre el evento emprendedor
(Shapero \& Sokol, 1982), recalca la combinación entre las percepciones de deseabilidad para emprender y las percepciones de dificultad para lograrlo.Y en la decisión de emprender, las claves motivacionales más frecuentes están asociadas a la autorrealización, a la independencia y a la necesidad de logro (Barba-Sánchez \& AtienzaSahuquillo, 2012).

Todo ello conecta directamente con el impacto del emprendimiento dentro del proyecto profesional-personal de cada individuo. Este se sitúa en un plano transversal y holístico, de confluencia entre lo vital y lo profesional, entre las necesidades más inmediatas y las metas de autorrealización (Patton \& McMahon, 2006). De hecho, diversas teorías enfatizan la importancia de la adaptabilidad de la carrera (Lent, 2012; Tolentino, García, Lu, Restubog, Bordia, \& Plewa, 2014), en vínculo con la noción de carrera flexible a lo largo de la vida (career across the lifecourse) (Tomlinson, Baird, Berg, \& Cooper, 2016). Y también se insiste en las conexiones entre las secuencias de la carrera profesional y otros aspectos de la vida de las personas, incluyendo sus roles familiares y otras actividades personales (Savickas, 2013; Super, 1990).

Desde el campo específico de la orientación profesional, junto con las competencias específicas del ámbito profesional y las de gestión empresarial, cabe considerar las competencias de gestión de la carrera profesional (Greenhaus, Callanan, \& Godshalk, 2010; Taveira, 2013), núcleo que conecta con las competencias de carácter transversal en el caso de personas emprendedoras. Se trata de competencias que implican componentes cognitivos, actitudinales y motivacionales asociados con la capacidad de planificación y de toma de decisión. Tienen por tanto un valor instrumental y estratégico en el desarrollo de la carrera profesional, se van renovando y ampliando a lo largo la trayectoria vital-profesional del individuo en función de sus circunstancias en cada momento (Jarvis, 2003; Lent, 2012).

El proyecto emprendedor debe formar parte y estar en coherencia con el proyecto vital y profesional de la persona. En ese marco, la acción formativa y orientadora habrá de adoptar enfoques más holísticos e integrales (Patton \& McMahon, 2006) y ayudar a optimizar los procesos de toma 
de decisiones a lo largo de la vida del individuo (Krumboltz, 1996). Cobran especial relevancia las competencias vinculadas al mantenimiento de una autoimagen positiva. Así en las teorías clásicas sobre el desarrollo profesional, el autoconcepto y los sentimientos de autoeficacia ocupan un espacio esencial en el desarrollo de cada individuo (Bandura, 1999; Super, 1990) por cuanto inciden directamente sobre las preferencias y están asociados al éxito profesional (Lent \& Brown, 1994). Asimismo, la dimensión axiológica que encaja los valores vitales de cada persona se ha identificado como un elemento esencial que otorga sentido a las decisiones y metas (Prioste, Narciso, \& Gonçalves, 2012; Krumboltz, 1996; Super, 1990).

Desde el enfoque cognitivo se habla incluso de la existencia de una inteligencia emprendedora, como conjunto de estrategias psicológicas que pone en funcionamiento un emprendedor para lograr el éxito en el negocio (Envick, 2014; Heller, 2010). De hecho, hay autores como Korotov, Khapova y Arthur (2011) que defienden la noción de carrera con espíritu emprendedor (career entrepreneurship), diferenciada de la carrera emprendedora (entrepreneural career), que consistente en usar un enfoque eminentemente empresarial sin prestar atención a la globalidad del plan vital.

Ambas teorías, social cognitiva (Bandura, 1999; Lent \& Brown, 1994) y de la construcción de la carrera (Savickas, 2013), evidencian la relevancia de la percepción de la autoeficacia en el éxito empresarial, entendida esta como la convicción de poder organizar y ejecutar efectivamente acciones para producir los resultados requeridos (Bandura, 1999). La necesidad de logro y el locus de control interno son rasgos de la persona emprendedora, a la vez que se le atribuye una actitud proactiva como conducta estratégica de anticipación que incluye la capacidad para representarse futuras situaciones o problemas, y la planificación a largo plazo (Ares, 2004). Es igualmente relevante la fuerte predisposición al cambio, a la innovación y a la creatividad, junto con el deseo de independencia personal y la capacidad de mantener la automotivación (Trejo López, 2015).

También se ha descrito la propensión a asumir riesgos y la mayor tolerancia a la ambigüedad y la incertidumbre, y un rasgo de mayor resiliencia, conductas de persistencia y de flexibilidad (PérezLeón, González-López, \& Rodríguez-Ariza, 2016), tomando un sentido práctico en el desempeño profesional. Otros estudios (Cardon, Gregoire, Stevens, \& Patel, 2013) han analizado la presencia de factores emocionales y cognitivos en el desarrollo del emprendedurismo, en particular la asertividad, la habilidad social para ejercer el liderazgo y el trabajo en equipo, junto con la autodisciplina, la tenacidad y el compromiso.

Este conjunto de competencias vinculadas al mundo empresarial y profesional confluye de manera significativa con las competencias clave asumidas en el ámbito de la orientación profesional y en el enfoque tradicional por competencias (Martínez \& Carmona, 2009).

El trabajo que se presenta tiene como finalidad diseñar un instrumento para evaluar las competencias que favorecen la carrera emprendedora y permiten gestionar los talentos que conducen al éxito en el mundo empresarial. Está enmarcado dentro del proyecto Diseño de la carrera y gestión del talento emprendedor ${ }^{l}$, cuya finalidad es implementar una estrategia motivadora para el autoempleo y formativa en la gestión de la carrera emprendedora. Se parte de la idea de que, independientemente de que sus comienzos hayan sido por vocación o por necesidad, sin duda los empresarios consolidados ponen en juego un conjunto de competencias estratégicas que les permiten sostener su actividad y articularla a su vez dentro de su proyecto vitalprofesional.

\section{Método}

\section{Objetivo}

Este trabajo está focalizado en los empresarios consolidados y en transición, en el contexto español, con la finalidad de explorar las competencias que favorecen el éxito de la carrera emprendedora. Para ello, se realiza un estudio de diseño y validación del instrumento denominado

\footnotetext{
${ }^{1}$ Financiado por el Ministerio de Economía y Competitividad en la convocatoria de 2013 Plan Estatal I+D 2013-2016 Excelencia, con Referencia: EDU2013-45704-P, Coord. Magdalena Suárez-Ortega, (Periodo de ejecución 2014-2017) Universidad de Sevilla, España. Web http://transitions.careers/
} 
Cuestionario de gestión de la carrera emprendedora (CGCE), cuyos objetivos son:

1. Diseñar y validar un cuestionario válido y fiable que permita evaluar las competencias que utilizan los empresarios/emprendedores para gestionar su carrera. Concretamente:

1.1.Las competencias estratégicas específicas que son implementadas para la gestión de su carrera emprendedora.

1.2. Las competencias generales de gestión de su carrera vital-profesional.

2. Explorar y caracterizar posibles grupos o perfiles competenciales en función de las respuestas al cuestionario.

\section{Procedimiento}

Sobre la base de un amplio rango de reactivos, el instrumento se ha ido depurando y reduciendo a través de varias fases. Primeramente, en una etapa exploratoria preliminar (basada en la revisión teórica y en la realización de entrevistas y grupos de discusión) se ha elaborado el primer borrador del instrumento. A continuación, (a) mediante la evaluación de expertos, se ha estudiado su validez de contenido; seguidamente, (b) se ha estudiado la fiabilidad, para luego (c) realizar un estudio de la estructura del instrumento, comprobando la fiabilidad de los factores y obteniendo así la versión final. Por último, se exploró la configuración de perfiles competenciales a partir de los factores obtenidos.

\section{Instrumento}

La revisión de la literatura científica permitió identificar un conjunto de categorías para configurar las grandes dimensiones del instrumento. Se han hallado otros instrumentos para evaluar la carrera emprendedora y sus distintas dimensiones (Cardon et al., 2013; Fiore, Niehm, Hurst, Son, \& Sadachar, 2013; Envick, 2014) que han servido como base inicial.Específicamente, en lo que respecta a las competencias emprendedoras, se ha considerado el modelo cognitivo de Envick, Entrepreneurial Intelligence Model (2014), el cual mantiene la relevancia de tres habilidades para el desarrollo del emprendimiento (pasión, visión y valor), las cuales se asocian a los comportamientos y actitudes presentes en el desempeño del emprendedor. En lo que se refiere a las competencias de gestión de la carrera, el diseño se ha basado en el modelo de Savickas sobre la conducta profesional (2013), el cual aporta elementos para comprender cómo las personas eligen y desarrollan un trabajo en conexión con aspectos vocacionales, qué habilidades ponen en juego y cómo las articulan para sentirse realizados y satisfechos vocacionalmente.

Con el fin de contrastar el marco teórico de partida, se llevó a cabo el estudio exploratorio preliminar de carácter cualitativo (Suárez Ortega, Cortés Pascual, \& Sánchez García, 2015) en el que se han efectuaron 15 entrevistas semiestructuradas (a técnicos que desarrollan programas de asesoramiento y técnicos de orientación a emprendedores) y 4 grupos de discusión en los que han participado empresarios consolidados y en transición. Este estudio previo permitió identificar categorías transversales, triangulando con las dimensiones procedentes de la revisión teórica. La versión inicial del cuestionario se estructuró en tres partes:

- Aspectos sociodemográficos (11 ítems), que permiten caracterizar a los encuestados: perfil emprendedor, comunidad autónoma, sexo, edad, entorno, cargas familiares, antigüedad del proyecto emprendedor, nivel de formación, presencia de discapacidad, sector de actividad y grado de innovación del negocio.

- Escala A de Competencias emprendedoras (42 ítems). Habilidades estratégicas asociadas a comportamientos, actitudes y personalidad presentes en el desempeño diario del emprendedor.

- Escala B de Competencias generales de gestión de la carrera (19 ítems). Competencias que posibilitan la toma de decisiones, la planificación y la implementación del proyecto vital y profesional.

Para la validación del contenido se recabó la valoración de 16 expertos con dos tipos de perfiles: 9 técnicos expertos en el ámbito del emprendimiento, y 7 investigadores universitarios del ámbito socio-educativo y de la orientación (Edad promedio de 38.2 años; 10 mujeres y 6 varones). El protocolo de evaluación recogió, sobre una escala de 1 a 6 , los criterios de pertinencia (adecuación para la dimensión que se desea medir) y de claridad (si la redacción no es confusa y permite una fácil comprensión), así como las apreciaciones y sugerencias de carácter 
cualitativo. Los resultados permitieron realizar una primera depuración del contenido del cuestionario en las dos escalas.

\section{Participantes}

El cuestionario se administró a 248 emprendedores/as (margen de error de $\pm 6.2 \%$ al nivel de confianza del 95\%), vía on-line a través del software Survey-monkey. La selección se realizó de forma incidental, atendiendo a criterios de equilibrio y diversidad sociodemográfica. Se distribuye del siguiente modo: Perfil consolidado (63.7\%)y perfil en transición (36.3\%); por sexos, el $55.6 \%$ son mujeres y el $44.4 \%$ varones; edades comprendidas entre 19 y 69 (promedio de 43.7 años); el 69.4\% desarrolla su actividad en el sector servicios, el $14.5 \%$ en actividades profesionales o artísticas, y el $11.7 \%$ en el sector agrario, la construcción o la industria; residentes en 17 comunidades autónomas españolas; yen cuanto al nivel de formación, el $61.7 \%$ tiene estudios superiores, el $36.7 \%$ estudios obligatorios o postobligatorios, y sólo el $1.6 \%$ no tiene estudios reglados.

\section{Análisis de datos}

En la fase de validación de contenido, la información proporcionada por los expertos fue analizada de forma descriptiva con medidas de tendencia central. Para estudiar la validación de constructo de ambas escalas se utilizó el análisis factorial exploratorio (AFE). Previa comprobación de la medida de adecuación muestral de Kaiser-Meyer-Olkin (KMO) y prueba de esfericidad de Bartlett. El modelo factorial resultante se sometió al análisis factorial confirmatorio (AFC) y la confiabilidad como consistencia interna de los factores obtenidos se verificó a través del índice alpha de Cronbach. Para el objetivo 2 se optó por el análisis de conglomerados (cluster) por ser una técnica multivariante que permite agrupar respuestas creando y diferenciando grupos con perfiles homogéneos en función de una serie de cualidades, empleando la representación gráfica (dendrograma) para interpretar las agrupaciones.

Los análisis estadísticos se efectuaron con el apoyo de tres softwares estadísticos: SPSS 22 (para descriptivos, AFE y consistencia interna),
IBM AMOS 22 (para el AFC) y SPAD 8.2.8 (para el análisis de clusters).

\section{Resultados}

\section{Validez de contenido}

En el marco del objetivo 1, la valoración de los expertos arroja resultados satisfactorios atendiendo a la media y particularmente a la mediana (Tabla 1), tanto en el criterio de pertinencia como en el de claridad. Se registran valoresligeramente inferiores en la dimensión de claridad frente a la de pertinencia.

Se han tenido en cuenta estos resultados, junto con las valoraciones cualitativas aportadas por los jueces de forma escrita, atendiendo especialmente a la necesidad de reducir su extensión y eliminar reiteraciones o superposiciones de contenido entre los ítems. Este análisis facilitó -junto con el estudio de validez de constructo y de fiabilidadla toma de decisiones para depurar y reducir el instrumento hasta configurar la versión final.

\section{Escala de Competencias Emprendedoras (CE): Validez de constructo y fiabilidad}

Para la escala CE (objetivo 1.1), se han comprobado las condiciones necesarias para la realización de AFE, encontrando que el índice KMO (.902) es muy elevado y la significación del Test de esfericidad de Bartlett $(p<.001)$ garantiza la existencia de suficientes intercorrelaciones entre las variables para que sea posible la factorización de las mismas.

Sobre los 34 ítems incluidos inicialmente, se han aplicado diversas pruebas de AFE con diferentes métodos de extracción y de rotación, sin obtener inicialmente resultados satisfactorios, dado que algunos ítems no contaban con adecuados valores de comunalidad en el factor. Tras un proceso de depuración hasta obtener una solución factorial satisfactoria, para el resultado final se establecieron 21 ítems optando por el método de extracción de Componentes Principales, con rotación ortogonal mediante Varimax. En esta solución factorial todos los ítems tienen buenas comunalidades y por tanto están bien representados en su factor. El AFE ha determinado la existencia de 4 factores que explican en total algo más del $59 \%$ de la 
Tabla 1. Pertinencia y claridad del cuestionario (Juicio de expertos)

\begin{tabular}{|c|c|c|c|c|c|c|c|c|}
\hline & \multirow{2}{*}{$\begin{array}{l}\text { Núm. } \\
\text { Ítems }\end{array}$} & \multirow{2}{*}{ Rango } & \multicolumn{3}{|c|}{ Pertinencia } & \multicolumn{3}{|c|}{ Claridad } \\
\hline & & & $M$ & $M d$ & $D T$ & $M$ & $M d$ & $D T$ \\
\hline \multirow{2}{*}{ Sociodemográficos } & & Min. & 4.81 & 6.00 & .000 & 4.81 & 5.50 & .000 \\
\hline & & Max. & 6.00 & 6.00 & 1.797 & 6.00 & 6.00 & 1.797 \\
\hline \multirow{2}{*}{$\begin{array}{l}\text { A. Competencias } \\
\text { emprendedoras }\end{array}$} & \multirow{2}{*}{25} & Min. & 5.31 & 6.00 & .000 & 5.25 & 5.50 & .250 \\
\hline & & Máx. & 6.00 & 6.00 & 1.544 & 5.94 & 6.00 & 1.486 \\
\hline \multirow{2}{*}{$\begin{array}{l}\text { B. Competencias } \\
\text { generales de gestión } \\
\text { de la carrera }\end{array}$} & \multirow{2}{*}{20} & Min. & 5.50 & 6.00 & .000 & 5.63 & 6.00 & .250 \\
\hline & & Máx. & 6.00 & 6.00 & 1.033 & 5.94 & 6.00 & 1.258 \\
\hline \multirow{2}{*}{ Global } & \multirow{2}{*}{53} & Min. & 4.81 & 6.00 & 1.797 & 4.81 & 5.50 & .000 \\
\hline & & Máx & 6.00 & 6.00 & .000 & 6.00 & 6.00 & 1.797 \\
\hline
\end{tabular}

Nota: Escala de 1 a 6 (1=Mínima pertinencia/claridad; 6=Máxima pertinencia/claridad)

variabilidad empírica observada. Todos los ítems presentan altas saturaciones (de al menos .600) en su factor, determinando con solidez su pertenencia al mismo. La Tabla 2 resume los resultados obtenidos.

Se han denominado y descrito los 4 factores obtenidos del siguiente modo:

- A1: Autoeficacia y proactividad. Configurado por 9 ítems $(2,3,4,5,6,8,9,10$ y 21), este factor recoge un conjunto de habilidades y actitudes, estratégicas y esencialmente proactivas, dirigidas a mantener un fuerte compromiso con su proyecto emprendedor.

- A2: Asertividad y control emocional. Integrado por 6 ítems $(17,18,20,27,32$ y 33) centrados en la implementación, en el día a día, de comportamientos emocionales que implican la identificación de las necesidades propias y de los clientes, la apertura a la cultura y la adaptación y ajuste constante de la actividad.

- A3: Liderazgo participativo. Se compone de 3 ítems $(24,30$ y 31$)$ que agrupan actitudes y competencias de liderazgo y de trabajo en equipo.

- A4: Afrontamiento de riesgos y dificultades. Agrupa otros 3 ítems (12, 13 y 16), vinculados a la asunción de riesgos y a la actitud resiliente frente a las situaciones desfavorables que tiene lugar en el desempeño profesional emprendedor.

La Figura 1 permite observar el modelo teórico a comprobar mediante el AFC, conformado por los 4 factores antes extraídos y los 21 ítems que los definen. Los números de las variables se corresponden con el número identificativo del ítem.

La Tabla 3 presenta el resumen de los estadísticos de ajuste utilizados. Se observa que el valor RMSEA se encuentra por encima del corte
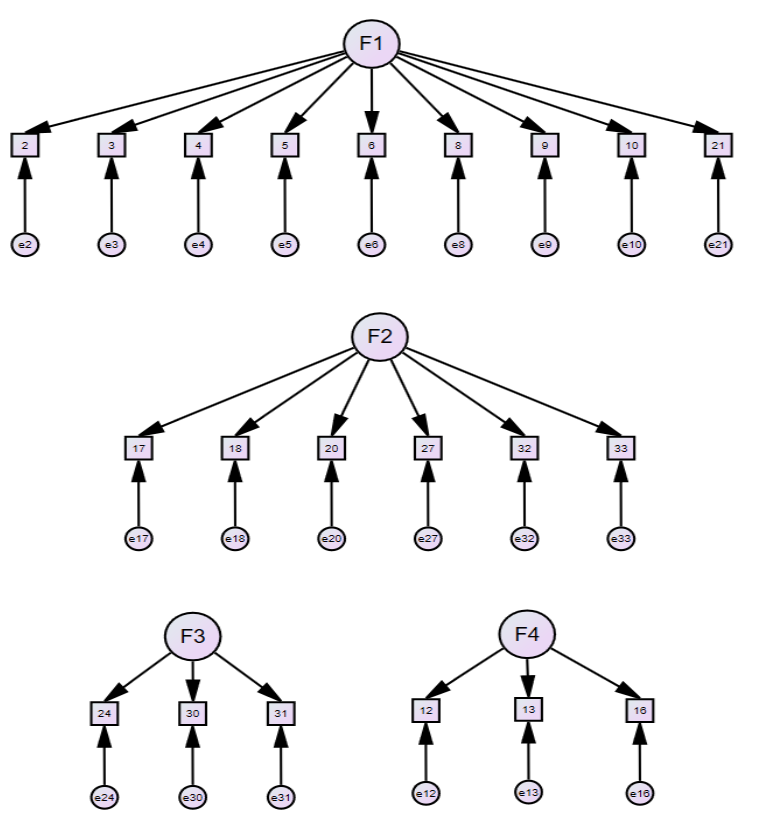

Figura 1: Diagrama de flujo del AFC. Subescalas de CE

.080 siendo su valor ligeramente superior dentro del IC (95\%): .083 - .100; por tanto apenas genera dudas sobre el ajuste de los datos al modelo, puesto que alguno de los demás índices sí superan el corte de .800 , situándose entre .744 y .812 ; y los que no superan el corte se encentran muy cerca del mismo.

Se estima que el resultado del AFC es fiable $\mathrm{y}$, consecuentemente, se considera que el ajuste del modelo teórico es suficiente. Se observa que los coeficientes estandarizados (Tabla 3), todos altamente significativos (al menos para $p<.01$ ) permiten establecer la asociación de los ítems con su factor esperado. En definitiva, los resultados confirman la pertenencia de todos los ítems al factor esperado, validando el modelo propuesto a contraste. 
Tabla 2. Análisis Factorial Exploratorio. Escala de CE

$\mathrm{KMO}=.902 ;$ Prueba de esfericidad de Bartlett: $\mathrm{Chi}^{2}=2183.609 ; \mathrm{gl}=210 ; p<.000$

\begin{tabular}{|c|c|c|c|c|c|c|}
\hline \multicolumn{2}{|c|}{$\begin{array}{l}\text { Componentes principales, rotación Varimax. Normaliz. Kaiser (6 } \\
\text { iteraciones) }\end{array}$} & \multicolumn{2}{|c|}{$\mathrm{F} 1$} & $\mathrm{~F} 2$ & F3 & $\mathrm{F} 4$ \\
\hline$\%$ Varianza total explicada & & & & 17.28 & 10.18 & 10.06 \\
\hline$\%$ Acumulado de varianza & & & & 39.03 & 49.20 & 59.27 \\
\hline \multirow{2}{*}{$\mathrm{N}^{\mathrm{o}}$ Ítem } & \multicolumn{2}{|c|}{ Descriptivos } & & \multicolumn{2}{|c|}{ Saturaciones $>.450$} & \\
\hline & Media & D.T. & F1 & F2 & F3 & F4 \\
\hline $\begin{array}{l}\text { 4-En general, soy optimista, relativizo los problemas y } \\
\text { considero los errores como oportunidades de aprender }\end{array}$ & 4.89 & 1.06 & .737 & & & \\
\hline $\begin{array}{l}\text { 8-Soy una persona apasionada con las cosas que hago, } \\
\text { normalmente me automotivo para avanzar y trabajar } \\
\text { en el día a día }\end{array}$ & 5.06 & 1.02 & .708 & & & \\
\hline $\begin{array}{l}\text { 5-Haga lo que haga, tengo fe y seguridad en mí } \\
\text { mismo/a y en que lo conseguiré }\end{array}$ & 4.73 & 1.01 & .703 & & & \\
\hline $\begin{array}{l}\text { 2-Soy una persona con iniciativa, me gusta introducir } \\
\text { cambios con frecuencia y agilidad (no estancarme), } \\
\text { aunque esto me haga salir de mi "zona de confort" }\end{array}$ & 4.84 & 1.18 & .666 & & & \\
\hline $\begin{array}{l}\text { 6-Suelo alternar momentos de estabilidad y momentos } \\
\text { de cambio en lo que hago }\end{array}$ & 4.32 & 1.11 & .659 & & & \\
\hline $\begin{array}{l}\text { 3-Soy constante en el tiempo y persistente cuando me } \\
\text { propongo una meta }\end{array}$ & 4.97 & 1.08 & .650 & & & \\
\hline $\begin{array}{l}\text { 10-Normalmente, me adapto con flexibilidad y } \\
\text { replanteo la estrategia cuando las cosas no salen como } \\
\text { esperaba }\end{array}$ & 4.77 & 1.01 & .643 & & & \\
\hline $\begin{array}{l}\text { 9-Generalmente soy resistente al estrés y a la tensión } \\
\text { que conlleva mi trabajo; mantengo la calma cuando } \\
\text { me enfrento a un problema difícil en mi empresa }\end{array}$ & 4.43 & 1.27 & .629 & & & \\
\hline $\begin{array}{l}\text { 21-Soy práctico/a, pienso en soluciones no en } \\
\text { problemas }\end{array}$ & 4.93 & 1.02 & .626 & & & \\
\hline $\begin{array}{l}\text { 33-Soy capaz de captar las necesidades de mis } \\
\text { clientes(as) e incorporarlas a mi proyecto empresarial }\end{array}$ & 4.98 & 0.92 & & .734 & & \\
\hline 18-Me implico a fondo en mis objetivos del día a día & 5.09 & 0.91 & & .722 & & \\
\hline $\begin{array}{l}\text { 27-Suelo tener en cuenta las normas sociales y } \\
\text { culturales (costumbres, tradiciones, etc.) de los } \\
\text { contextos de mi actividad empresarial }\end{array}$ & 4.81 & 1.11 & & .704 & & \\
\hline $\begin{array}{l}\text { 32-Me gusta tomarme el tiempo necesario para } \\
\text { escuchar y comprender a mis clientes(as) y personas } \\
\text { con las que trabajo }\end{array}$ & 5.14 & 0.94 & & .693 & & \\
\hline $\begin{array}{l}\text { 17-El día a día me hace ser consciente de que siempre } \\
\text { hay algo que aprender }\end{array}$ & 5.39 & 0.85 & & .678 & & \\
\hline $\begin{array}{l}\text { 20-Me gusta hacer planificaciones semanales, ir } \\
\text { adaptando mi plan estratégico día a día, de acuerdo } \\
\text { con las necesidades y circunstancias que van surgiendo }\end{array}$ & 4.33 & 1.36 & & .600 & & \\
\hline $\begin{array}{l}\text { 30-Prefiero ejercer un liderazgo compartido con } \\
\text { personas con las que me siento compenetrado/a }\end{array}$ & 4.74 & 1.26 & & & .803 & \\
\hline $\begin{array}{l}\text { 31-Al ejercer el liderazgo, me parece fundamental } \\
\text { rodearme de personas con mucho talento }\end{array}$ & 4.95 & 1.22 & & & .688 & \\
\hline $\begin{array}{l}\text { 24-Me gusta trabajar en equipo con el personal de mi } \\
\text { empresa }\end{array}$ & 4.83 & 1.30 & & & 630 & \\
\hline $\begin{array}{l}\text { 13-Los importantes riesgos que a menudo debo } \\
\text { afrontar en mi empresa me provocan mucho estrés }\end{array}$ & 3.46 & 1.37 & & & & .801 \\
\hline $\begin{array}{l}\text { 12-Me cuesta afrontar la incertidumbre y los } \\
\text { problemas imprevistos }\end{array}$ & 3.14 & 1.41 & & & & .757 \\
\hline $\begin{array}{l}\text { 16-El temor a fracasar en mi empresa es algo que } \\
\text { siempre está presente }\end{array}$ & 3.26 & 1.54 & & & & .745 \\
\hline
\end{tabular}

Se ha estudiado la fiabilidad de la escala completa $(\alpha=.868)$ y de cada una de las subescalas correspondientes a las dimensiones definidas por los factores. Los resultados permiten comprobar la existencia de una muy buena fiabilidad $(p=.000)$ tanto en el F1 $(\alpha=.887)$ como en el F2 ( $\alpha=.819)$, los cuales influyen activamente en la también elevada fiabilidad de la escala completa. Y los factores F3 (.702) y F4 ( $\alpha=.701)$ alcanzan buenas fiabilidades a pesar de su escasa longitud. 
Tabla 3. Análisis Factorial Confirmatorio. Escala CE

\begin{tabular}{|c|c|c|c|c|c|c|c|}
\hline \multicolumn{2}{|c|}{$\begin{array}{l}\text { Modelo } \\
\text { Cuatro factores }\end{array}$} & & $\begin{array}{c}\text { RMSEA } \\
.091\end{array}$ & $\begin{array}{l}\text { NFI } \\
.744\end{array}$ & $\begin{array}{l}\text { IFI } \\
.812\end{array}$ & $\begin{array}{l}\text { TLI } \\
.765\end{array}$ & $\begin{array}{l}\text { CFI } \\
.808 \\
\end{array}$ \\
\hline \multirow{2}{*}{$\mathrm{N}^{\mathrm{o}}$ Ítem } & \multicolumn{4}{|c|}{ Coef. de regresión estandarizados } & \multirow{2}{*}{\multicolumn{3}{|c|}{ Ecuación estructural }} \\
\hline & Factor 1 & Factor 2 & Factor 3 & Factor 4 & & & \\
\hline 2 & .614 & & & & $\mathrm{~F} 1=$ & $84+0$. & It2 \\
\hline 3 & .653 & & & & $\mathrm{~F} 1=$ & $97+0$. & $<\mathrm{It} 3$ \\
\hline 4 & .735 & & & & $\mathrm{~F} 1=$ & $89+0$ & < It4 \\
\hline 5 & .744 & & & & $\mathrm{~F} 1=$ & $73+0$ & It5 \\
\hline 6 & .513 & & & & $\mathrm{~F} 1=$ & $32+0.5$ & < It6 \\
\hline 8 & .793 & & & & $\mathrm{~F} 1=$ & $06+0.8$ & $<\mathrm{It} 8$ \\
\hline 9 & .661 & & & & $\mathrm{~F} 1=$ & $43+0.8$ & $<$ It9 \\
\hline 10 & .747 & & & & $\mathrm{~F} 1=$ & $6+0.7$ & It10 \\
\hline 21 & .742 & & & & $\mathrm{~F} 1=$ & $3+0.7$ & It 21 \\
\hline 17 & & .703 & & & $\mathrm{~F} 2=$ & $9+0.6$ & It17 \\
\hline 18 & & .756 & & & $\mathrm{~F} 2=$ & $9+0.6$ & It 18 \\
\hline 20 & & .478 & & & $\mathrm{~F} 2=$ & $3+0.6$ & It20 \\
\hline 27 & & .642 & & & $\mathrm{~F} 2=$ & $1+0.7$ & It 27 \\
\hline 32 & & .754 & & & $\mathrm{~F} 2=$ & $4+0.7$ & It32 \\
\hline 33 & & .756 & & & $\mathrm{~F} 2=$ & $8+0.6$ & It 33 \\
\hline 24 & & & .635 & & $\mathrm{~F} 3=$ & $3+0.8$ & It 24 \\
\hline 30 & & & .747 & & $\mathrm{~F} 3=$ & $4+0.9$ & It 30 \\
\hline 31 & & & .612 & & $\mathrm{~F} 3=$ & $6+0.7$ & It 31 \\
\hline 12 & & & & .671 & $\mathrm{~F} 4=$ & $4+0.9$ & It 12 \\
\hline 13 & & & & .715 & $\mathrm{~F} 4=$ & $6+0.9$ & It13 \\
\hline 16 & & & & .610 & $\mathrm{~F} 4=$ & $6+0.9$ & It16 \\
\hline
\end{tabular}

\section{Escala de Competencias de Gestión de la Carrera (CGC):Validez de constructo y fiabilidad}

En el caso de laescala CGC (objetivo 1.2) se comprueba igualmente que se cumplen satisfactoriamente las condiciones necesarias para la realización de un AFE. El valor de KMO (.902) es muy elevado y la significación del Test de esfericidad de Bartlett $(p<.000)$ garantiza igualmente las condiciones para proceder al proceso de factorización de las mismas.

La aplicación de diversas pruebas de AFE permitió depurar la escala que pasó de 15 a 12 ítems. Sobre esta solución se aplicaron diferentes métodos de extracción y de rotación, aportando soluciones similares y en todos los casos los ítems cuentan con adecuados valores de su comunalidad. Para el resultado final se aplica el método de extracción de Componentes Principales, con rotación Promax, solución que maximiza las saturaciones de los ítems dentro de las dimensiones (Tabla 4). El resultado final (Tabla 5) determina la existencia de 2 factores con
6 ítems en cada uno de ellos (12 en total) que explican casi un $60 \%$ de la variabilidad observada; si bien cabe destacar que el primero de ellos, ya explica un $49 \%$ de la misma. Igualmente, la correlación entre ambos factores es muy elevada (.651) y todos los ítems presentan fuertes saturaciones (>.450) en uno de los dos factores, lo que garantiza claramente su pertenencia solo a uno de ellos.

Las dimensiones correspondientes a estos dos factores obtenidos son:

- B1: Competencias de toma de decisiones. Compuesto por 6 ítems $(35,36,37,38,48$ y 49) centrados en la toma de decisiones de carrera y la planificación desde el autoconocimiento y la coherencia con los propios valores.

- B2: Competencias de gestión del proyecto vitalprofesional. Integrado por 6 ítems (42, 43, 44, 45, 46 y 47) vinculados a una dimensión eminentemente práctica de la gestión de la carrera, en coherencia con las metas asumidas en el proyecto vital y profesional.

La Figura 2 muestra el modelo teórico que se desea probar con el AFC, con los 6 ítems 
Tabla 4. Análisis Factorial Confirmatorio. Escala CGC

$\mathrm{KMO}=.902$; Prueba de esfericidad de Bartlett: $\mathrm{Chi}^{2}=1394.705 ; \mathrm{gl}=66 ; \mathrm{p}=.000$

Componentes Principales, rotación Promax Normaliz. Kaiser (3 iteraciones)

$\%$ Varianza total explicada

$\%$ Acumulado de Varianza

$\mathrm{N}^{\mathrm{o}}$ Ítem

Descriptivos

Media

D.E

37-Me anticipo a posibles dificultades antes de tomar una

decisión profesional o en mi vida personal

\begin{tabular}{|c|c|c|c|}
\hline 4.63 & 1.00 & .917 & \\
\hline 4.86 & 1.01 & .758 & \\
\hline 4.79 & 0.97 & .742 & \\
\hline 4.87 & 1.06 & .729 & \\
\hline 4.50 & 1.06 & .711 & \\
\hline 4.29 & 0.92 & .461 & \\
\hline 4.52 & 1.07 & & .940 \\
\hline 4.51 & 1.07 & & .880 \\
\hline 4.88 & 0.91 & & .677 \\
\hline 4.74 & 1.03 & & .634 \\
\hline 4.75 & 1.06 & & .542 \\
\hline 4.45 & 1.07 & & .481 \\
\hline F1 & & -- & .651 \\
\hline $\mathrm{F} 2$ & & .651 & -- \\
\hline
\end{tabular}

realista

48-Considero todas las posibilidades y experiencias previas para

tomar decisiones importantes

49-Mi proyecto empresarial es totalmente coherente con mis

metas y objetivos personales

36-La información que tengo sobre mí mismo/a me sirve para

elaborar y desarrollar mi proyecto empresarial

35-Tengo un excelente concepto de mí mismo/a

44-Soy capaz de detectar mis nuevas necesidades para mejorar

mi vida personal y profesional

45-Suelo darme cuenta cuando surgen oportunidades

interesantes y sé aprovecharlas

46-Tengo en cuenta mis puntos débiles y saco el máximo partido

de mis puntos fuertes (cualidades, formación, experiencias, etc.)

en la gestión de mi proyecto empresarial

42-Tengo claros los roles profesionales que me interesa

desempeñar dentro de mi empresa

47-Sé muy bien hacia dónde deseo dirigir mi futuro profesional

y mi vida en general

43-Afronto de forma equilibrada mis fracasos y mis éxitos

Saturaciones $>.450$

$\mathrm{F} 1$

F2

$49.18 \quad 8.78$

$49.18 \quad 59.97$

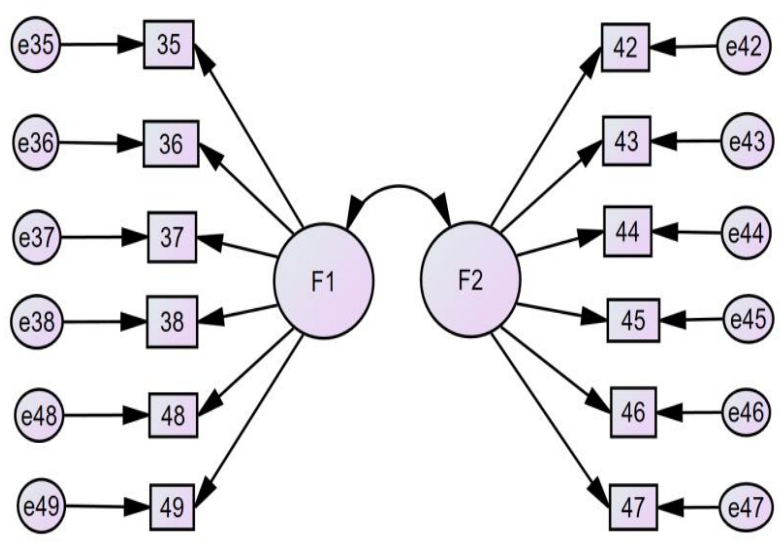

Figura 2. Diagrama de flujo del AFC. Escala CGC

relacionados respectivamente en 2 dimensiones correlacionadas entre sí. Los números de las variables, se corresponden con el número identificativo del ítem.

Como puede observarse en la tabla 5, el valor RMSEA no se encuentra por debajo de corte .080 siendo su valor algo superior dentro del IC (95\%): .091 - .122; aunque podría generar alguna duda sobre el ajuste de los datos al modelo, sin embargo, el resto de índices supera el corte de .800 sobradamente. Ante estos resultados se considera que el ajuste del modelo teórico con los datos empíricos es adecuado, de manera que el resultado del AFC es fiable. Todos los coeficientes estandarizados son altamente significativos $(p<.001)$, por lo que prueban la certeza de la asociación de los ítems con su factor esperado. Así mismo, se ha comprobado la muy elevada correlación entre ambas dimensiones.

Respecto a la fiabilidad de los factores, los valores obtenidos indicanuna muy buena $\mathrm{y}$ elevada fiabilidad $(p=.000)$, tanto de la escala completa con los 12 ítems $(\alpha=.905)$ como de ambas subescalas $(\alpha=.843$ para $F 1 ; \alpha=.852$ para F2).

\section{Análisis de conglomerados}

Para el objetivo 2 se ha efectuado el análisis de conglomerados a través de un método mixto, que combina inicialmente un método de agrupación (k-medias) con un método jerárquico (con el apoyo del software estadístico 
Tabla 5. Análisis Factorial Confirmatorio. Escala CGC

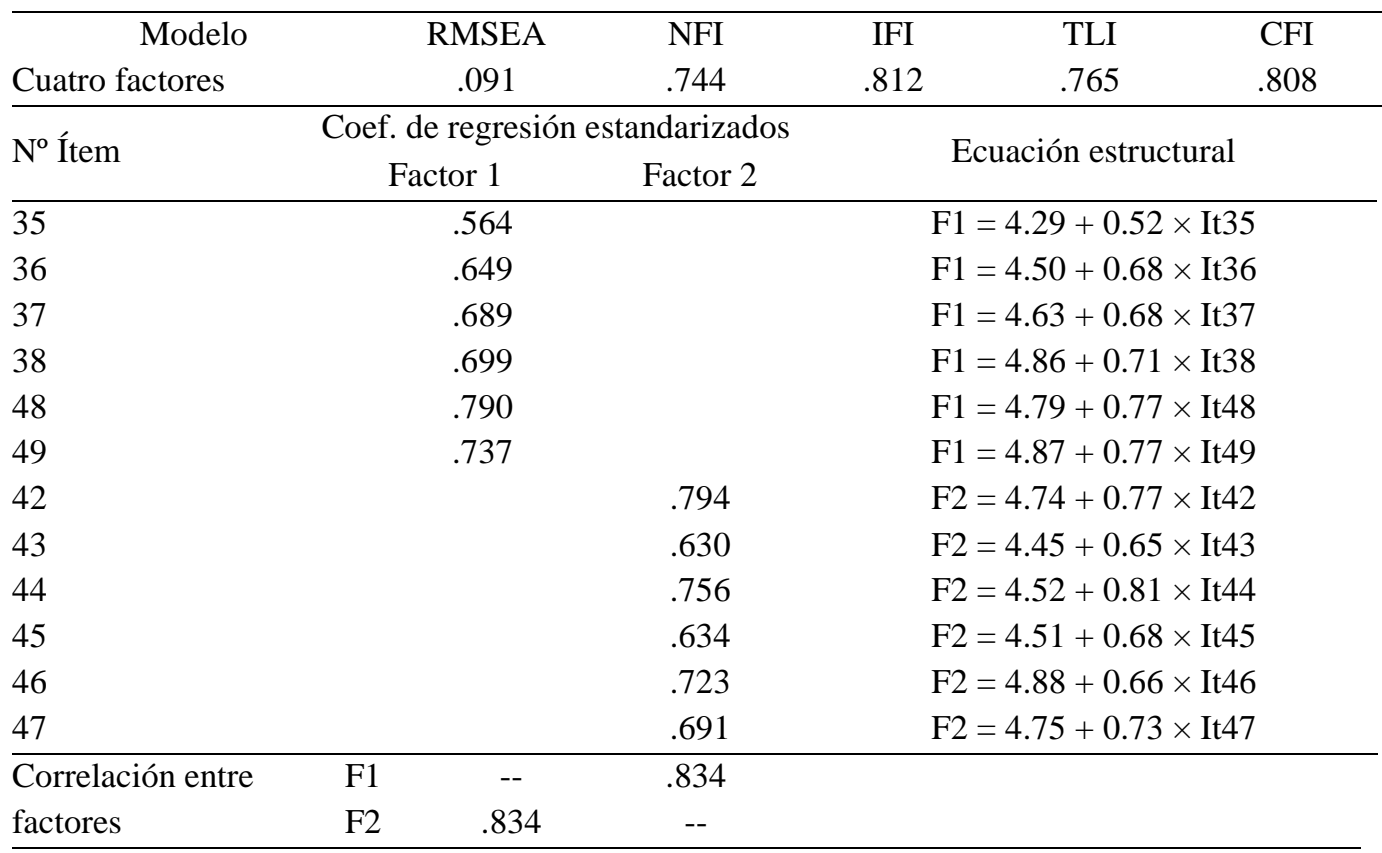

Tabla 6. Análisis de conglomerados. Caracterización de variables y categorías en los grupos

\begin{tabular}{|c|c|c|c|c|}
\hline Conglo-merados & $\begin{array}{l}\text { Variables en los } \\
\text { conglomerados }\end{array}$ & $\begin{array}{l}\text { Media } \\
\text { Cluster }\end{array}$ & $\begin{array}{l}\text { Categorías en los } \\
\text { conglomerados }\end{array}$ & $\%$ en grupo \\
\hline \multirow{7}{*}{$\begin{array}{l}1 \\
n=127 \\
51.21 \%\end{array}$} & $(+)$ Perfil consolid $* * *$ & 1.000 & $(+)$ Sin discap** & 87.402 \\
\hline & (+)B2-Ges.prác.proy*** & 0.391 & $(+)$ Entorno urba** & 88.189 \\
\hline & $(+)$ A2-control emoc $* * *$ & 0.289 & $(+)$ Total vinc.form. ${ }^{*}$ & 60.630 \\
\hline & (-)A1-Autoef.proac**** & 0.266 & (-)Con discap** & 12.598 \\
\hline & (+)A3-Lider partic*** & 0.217 & $(-)$ Entorno virtual $* * *$ & 2.362 \\
\hline & $(+)$ Mayor edad*** & 45.260 & & \\
\hline & (-)A4-Afron.dific* & -0.140 & & \\
\hline & $(+)$ A4-Afron.dific $* * *$ & 0.209 & $(+)$ Entorno virtual $* * *$ & 18.750 \\
\hline & (+)B1-Planif.carrera* & 0.194 & $(+)$ Con discap** & 31.250 \\
\hline 2 & $(+)$ Menor edad*** & 38.713 & $(+)$ Nivel form. sup* & 41.250 \\
\hline$n=80$ & (-)Perfil transición*** & 0.000 & (-)Sector act.prof.* & 7.500 \\
\hline \multirow[t]{3}{*}{$32.26 \%$} & & & (-)Nada innov* & 11.250 \\
\hline & & & $(-)$ Sin discap** & 68.750 \\
\hline & & & (-)Entorno urba** & 70.000 \\
\hline \multirow{6}{*}{$\begin{array}{l}3 \\
n=41 \\
16.53 \%\end{array}$} & (-)A1-Autoef.proac*** & -0.649 & (+)Nada innov*** & 41.463 \\
\hline & (-)B1-Planif.carrera*** & -0.689 & $(+)$ Sin vinc. form $* *$ & 21.951 \\
\hline & $(-)$ A3-Lider partic $* * *$ & -0.867 & (+)Form.posoblig* & 41.463 \\
\hline & (-)A2-Contr.emoc $* * *$ & -1.114 & (-)Total vinc. form* & 36.585 \\
\hline & (-)B2-Gesprác.proy*** & -1.320 & $(-)$ Form. sup ${ }^{* *}$ & 14.634 \\
\hline & & & (-)Algo innov** & 34.146 \\
\hline
\end{tabular}

Nota:(+)=Alto nivel; (-)=Bajo nivel; $* p<.05 ; * * p<.01 ; * * * p<.001$ 
SPAD 8.2.8). Se identifican tres grupos en función de los factores obtenidos en el estudio anterior. A continuación se ha realizado una caracterización de los perfiles de estos grupos, tanto en una variables categóricas (chi cuadrado, $p=.05$ ), como en variables cuantitativas (ANOVA) (Tabla 6 y Gráfico 3).

El dendrograma resultante (Figura 3) muestra claramente la existencia de tres conglomerados, obtenidos en función de las respuestas en las CE (autoeficacia y proactividad; asertividad y control emocional; estilo de liderazgo y afrontamiento de riesgos y dificultades), las CGC (planificación de la carrera y gestión proyecto profesional) y el perfil emprendedor.

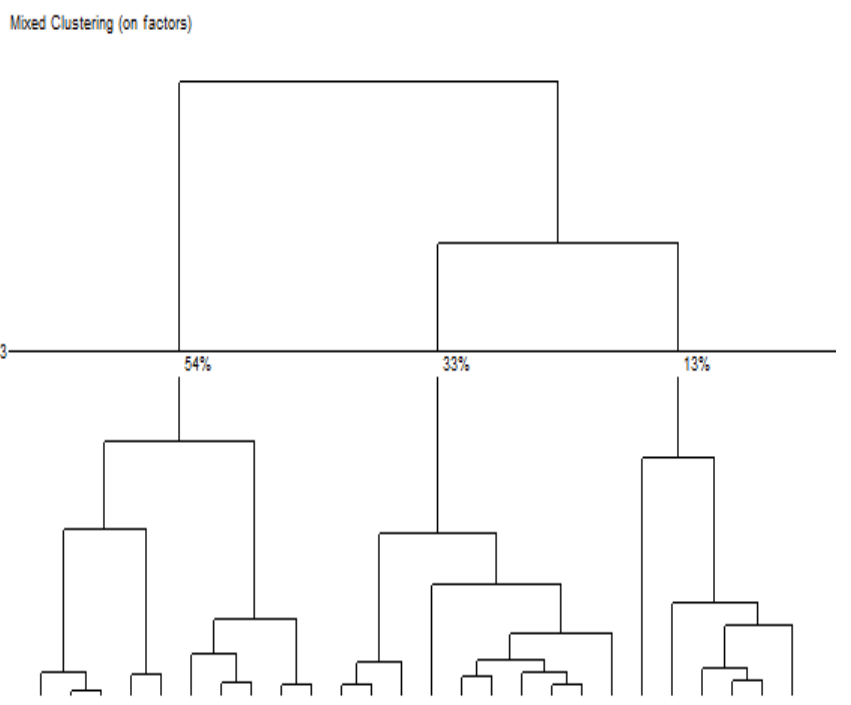

Figura 3: Dendrograma del análisis de conglomerados

Una vez obtenidos los 3 grupos, se realizó una caracterización de sus perfiles en función de variables del cuestionario. En general los resultados permiten asociar las clases a diferentes niveles de experiencia (perfiles de emprendimiento), percepciones sobre necesidades y sobre el éxito/fracaso profesional.

\section{Discusión y Conclusiones}

Como primera conclusión relativa al objetivo 1, puede afirmarse que el Cuestionario CGCE presenta buenas propiedades psicométricas. El AFE ha permitido completar la depuración de las dos escalas previamente validadas en su contenido a través de juicio de expertos, obteniendo estructuras

factoriales

satisfactorias estadísticamente. A su vez se confirman los modelos factoriales obtenidos a través del AFC y ambas escalas alcanzan una confiabilidad alta, tanto globalmente como en todas sus subescalas. Estas resultan en conjunto y, de acuerdo con la revisión de la literatura, teóricamente coherentes y en general coincidentes con los hallazgos de otros estudios. Y encajan con lo que otros autores (Heller, 2010; Envick, 2014) vienen denominando como inteligencia emprendedora.

El primer factor de la escala CE, referido al núcleo competencial de autoeficacia $y$ proactividad (A1), confirma su relevancia al encontrarse altos niveles entre los encuestados. El hallazgo de este factor indica la existencia de un nexo entre los rasgos de autoeficacia y proactividad, ya que agrupa habilidades vinculadas a conductas y rasgos de automotivación y autoconfianza, de propensión al cambio, la innovación y la iniciativa;de persistencia, flexibilidad, así como de previsión de necesidades. En definitiva, confluyen con un sentido práctico y proactivo en el desempeño profesional, de manera acorde y comprometida con el proyecto emprendedor. En este sentido, cuanto más confíe la persona en sus propios recursos y capacidades, mejor planificará y desarrollará su actividad emprendedora, favoreciendo así el locus de control interno (Trejo López, 2015).

Respecto al segundo factor competencial encontrado, asertividad y control emocional (A2), está integrado por competencias que puntúan con valores altos. Admiten la práctica cotidiana de comportamientos emocionales asertivos hacia los demás y hacia uno mismo, de implicación en las tareas, junto con una comprensión del entorno cultural, de forma adaptativa y ajustada a las necesidades, a la vez que invirtiendo energía. Algunos estudios (Sánchez \& Robles, 2014) asimismo han descrito la relación de las competencias emocionales con la autoeficacia emprendedora y también con la capacidad de afrontar las situaciones adversas (resiliencia) e incluso para automotivarse.

El tercer factor hallado de liderazgo participativo (A3) implica, de acuerdo con Lussier y Achua (2008) unas habilidades para influir en las personas del entorno laboral creado, 
para contar con su talento, lo cual no se opone a poseer un marcado sentido de la dirección para su organización (Lussier \& Achua, 2008). Las respuestas analizadas muestran niveles altos en esta competencia entre los emprendedores encuestados. Esto contrasta con los resultados de otros estudios que encuentran bajos niveles de competencias para el liderazgo y el trabajo en equipo entre los emprendedores (González Millán \& Parra Penagos, 2008; Trejo López, 2015). Lo cual invita a considerar la importancia de esta dimensión en lo que respecta a los programas de formación y orientación para el emprendimiento, de manera que se impulsen liderazgos capaces de generar cambios, de encontrar nuevas ideas y cauces de desarrollo.

Respecto al cuarto y último factor competencial identificado en la primera escala (CE), afrontamiento de riesgos y dificultades, los resultados aportan niveles intermedios $\mathrm{o}$ moderados de competencia. Si bien esto contribuye a confirmar, en coincidencia con otros estudios (Trejo López, 2015; Shapero \& Sokol, 1982), la presencia de este rasgo competencial (capacidad para asumir riesgos, para afrontar situaciones inciertas o desfavorables de forma resiliente) entre los emprendedores, sin embargo en nuestro caso no resulta tan marcado.

En lo que se refiere a la escala de competencias generales de gestión de la carrera (CGC), se obtienen valores promedio de nivel medio y medio-alto, lo que presenta implicaciones a tener en cuenta a la hora de establecer acciones de orientación. De alguna forma, se aprecia que en la gestión de la carrera, operan una habilidad de carácter más cognitivo-reflexivo sobre las propias metas y decisiones, y otra de carácter más práctico y aplicado en las situaciones de gestión de la carrera. La correlación encontrada en el modelo confirmado mediante AFC refleja una interdependencia de ambos núcleos competenciales, que deben funcionar en equilibrio y coherencia.

El análisis de perfiles competenciales (conglomerados) en relación con los factores hallados, ha permitido identificar y caracterizar tres grupos:

Un primer grupo $(n=127,51.21 \%)$ que constituye una clase de emprendedores con perfil consolidado, caracterizada por tener mayor edad, experiencia y éxito profesional, y que destacan por una óptima gestión práctica del proyecto profesional (B2), un alto control emocional (A2), una elevada autoeficacia/proactividad (A1), y que practican de forma marcada un liderazgo participativo (A3). Este grupo aparece sin embargo asociado a un bajo afrontamiento de las dificultades/resiliencia. Se agrupa con variables sociodemográficas que puntúan de manera positiva y significativa, situándose prioritariamente en el entorno urbano, presentando una vinculación entre la formación y su actividad empresarial y no tienen ninguna discapacidad. La vinculación de rasgos confluyentes en este perfil es consistente con estudios como el de Tolentino et al. (2014) que encontraron una relación entre la autoeficacia emprendedora, la adaptabilidad de la carrera y las intenciones emprendedoras, relación más marcada cuando existe experiencia previa en empresa familiar.

El segundo grupo $(n=80,32.26 \%)$ está integrado por emprendedores más jóvenes y en transición a la carrera emprendedora, que comparte con el grupo anterior una óptima planificación de la carrera, pero que contrasta con este presentando un alto afrontamiento de las dificultades/resiliencia, al tiempo que se asocia con un bajo nivel de experiencia profesional. La función discriminante extraída del análisis permite asimismo establecer diferencias significativas respecto al desarrollo de su actividad en un entorno virtual, destacando en su nivel de formación frente a los otros dos grupos con estudios superiores y presentando alguna discapacidad. Esta vinculación de la edad y otros factores de exclusión con la resiliencia se confirma en otros estudios que apoyan la idea de que es un rasgo sujeto a un proceso dinámico y evolutivo (Sánchez-Teruel \& Robles-Bello, 2015).

Por su parte, el tercer grupo $(n=41,16.53 \%)$ está representado por una combinación de ambos perfiles de emprendimiento (consolidado y en transición), y se caracteriza por niveles bajos en todos los núcleos competenciales (excepto el relativo al afrontamiento de dificultades/resiliencia). A su vez, se aprecian valores significativos en variables sociodemográficas, destacando que son personas con nivel formativo medio (estudios posobligatorios) pero su formación no es 
coherente con la actividad empresarial (en contraposición con el grupo 1) y el negocio creado no es nada innovador. Como puede apreciarse, este es el grupo que comparte mayores necesidades en el desarrollo de sus competencias de gestión de la carrera emprendedora.

En relación con el objetivo 2, la variable género no introduce diferencias significativas, por lo que hombres y mujeres se distribuyen de forma semejante en los tres grupos. Esto contrasta con otros estudios que encontraron diferencias, especialmente en rasgos como la autoeficacia y diversos aspectos del control emocional y las habilidades sociales (Ares, 2004; Trejo López, 2015). Asimismo, no introducen diferencias significativas el entorno geográfico de residencia, ni tampoco las cargas familiares.

El contraste de los dos primeros grupos revela que el perfil de experiencia emprendedora en el que está presente un factor generacional (edad), junto con el nivel e idoneidad de la formación, se presentan como factores relevantes asociados a los niveles competenciales de gestión de la carrera emprendedora. Esta conclusión es coherente con diversos estudios (GEM, 2014; Trejo López, 2015), que muestran rasgos significativamente diferentes entre los perfiles de empresarios consolidados y de emprendedores en transición.

Complementariamente, los resultados permiten inferir ciertas implicaciones en lo que respecta a las necesidades de desarrollo competencial. El primer grupo, aunque muestra altas competencias para gestionar su carrera emprendedora, presenta necesidades respecto a su capacidad de afrontamiento de dificultades y al desarrollo de un comportamiento resiliente en su campo profesional. Los integrantes del segundo grupo, sin embargo, aunque confían más en su disposición para afrontar riesgos e incertidumbres $\mathrm{y}$ en sus habilidades para planificar con coherencia su proyecto profesional, requieren desarrollar más aquellas competencias que les permiten desarrollarlo en la práctica. Y respecto al tercer grupo, se autopercibe de forma más negativa $\mathrm{y}$ menos optimista respecto a las posibilidades de emprendimiento, presentando más necesidades en cuanto todos los núcleos competenciales tanto generales para la planificación y desarrollo de su proyecto profesional, como específicamente para afrontar la carrera emprendedora.

En definitiva, el estudio contribuye aproporcionar un instrumento de evaluación con criterios fiables a la hora de diagnosticar las necesidades de las personas emprendedoras. Si bien es preciso considerar las limitaciones del estudio, entre ellas las que afectan a toda medida de autoinforme y las inherentes a la no aleatoriedad de la muestra. Consecuentemente, se hace necesario avanzar y profundizar en la investigación sobre estos ámbitos competenciales, e igualmente es aconsejable utilizar el instrumento en un proceso diagnóstico que integre estrategias complementarias.

Otra implicación práctica del estudio es su aplicación en el diseño de programas formativos y de asesoramiento al emprendedor. En este sentido, corrobora la idea de que la formación para el emprendimiento va más allá del asesoramiento en términos económicos, y requiere de un modelo holístico y de un enfoque por competencias (Patton \& McMahon, 2006; Savickas, 2013) que integre acciones personalizadas de naturaleza orientadora muy centradas en el desarrollo y gestión de la carrera emprendedora, desde el análisis pormenorizado de los perfiles competenciales.

\section{Referencias}

Ajzen, I. (1991). The theory of planned behavior. Organizational Behavior \& Human Decision Processes, 50, 179-211. doi:10.1016/07495978(91)90020-T

Ares, A. (2004). La conducta proactiva de los emprendedores. Portularia, 4, 493-498. doi: 10.5209/rev_ESMP.2013.v19.42151

Bandura, A. (1999). Social cognitivetheory of personality. En L. Pervin y O. John (Eds.), Handbook of Personality (2a Ed.) (pp.154196). Nueva York: Guilford.

Barba-Sánchez, V., \& Atienza-Sahuquillo, C. (2012). Entrepreneurial behavior: Impact of motivation factors on decision to create a new venture. Investigaciones Europeas de Dirección y Economía de la Empresa, 18, 32138.doi: 10.1016/S1135-2523(12)70003-5 
Cardon, M. S., Gregoire, D. A., Stevens, C. E., \& Patel, P. C. (2013). Measuring entrepreneurial passion: Conceptual foundations and scale validation. Journal of Business Venturing, 28(3), 373-396.doi: http://dx.doi.org/10.1016 /j.jbusvent.2012.03.003

Envick, B. R. (2014). Achieving entrepreneurial success through passion, vision \& courage: A cognitive model for developing entrepreneurial intelligence. Academy of Entrepreneurship Journal, 20(1), 55-74.

Fiore, A. M., Niehm, L. S., Hurst, J. L., Son, J., \& Sadachar, A. (2013). Entrepreneurial marketing: Scale validation with small, independently-owned businesses. Journal of Marketing Development and Competitiveness, 7(4), 63-86.

Global Entrepreneurship Monitor (2014).Informe GEM España 2014. Editorial Universidad de Cantabria y Asociación RED GEM y CISE.

Gómez-Araujo, E., Lafuente, E., Vaillant, Y., \& Gómez Núñez, L. M. (2015). El impacto diferenciado de la autoconfianza, los modelos de referencia y el miedo al fracaso sobre los jóvenes emprendedores. Innovar, 25(57), 157174. doi: 0.15446/innovar.v25n57.50358

González Millán, J. J., \& Parra Penagos, C. O. (2008). Caracterización de la cultura organizacional. Clima organizacional, motivación, liderazgo y satisfacción de las pequeñas empresas del valle de Sugamuxi y su incidencia en el espíritu empresarial. Pensamiento \& gestión, 25, 40-57.

Greenhaus, J. H., Callanan, G. A., \& Godshalk, V. M. (2010). Career management (4 ed.). ThousandOaks, California: Sage Pub., Inc.

Heller, L. (2010). Mujeres emprendedoras en AméricaLatina y el Caribe: Realidades, obstáculos y desarrollo. Santiago de Chile: CEPAL.

Jarvis, P. S. (2003). Career management skills. Keys to great career and a great life. Otawa, Onta rio: Canadian Career Development Foundation.

Korotov, K., Khapova, S. N. \& Arthur, M. B. (2011). Career entrepreneur ship. Organizational Dynamics, 40(2), 127-135.

Krumboltz, J. D. (1996). A social learning theory of career counseling. Stanford, Ca: Stanford University Press.
Lent, R. W. (2012). Career-life preparedness: Revisiting career planning and adjustment in the New Workplace. The Career Development Quarterly, 61(1), 2-14. doi: 10.1002/j.21610045.2013.00031.x

Lent, R. W., \& Brown, S. D. (1994). Social cognitive approach to career development: An overview. The Career Development Quarterly, 44, 310- 321.

Lussier, N. R., \& Achua, F.C. (2008). Liderazgo, aplicación, desarrollo y habilidades. México, D.F.: CengageLearning Editores.

Martínez, F. M., \& Carmona, G. (2009). Aproximación al concepto de "Competencias emprendedoras": valor social e implicaciones educativas. Revista Iberoamericana sobre Calidad, Eficacia y Cambio en Educación, 7(3), 82-98.

Patton, W., \& McMahon, M. (2006). Career Development and Systems Theory. Connecting Theory and Practice. Rotterdam: SensePublishers.

Pérez-León, M. C., González-López, M. J., \& Rodríguez-Ariza, L. (2016). Competencies for entrepreneurship as a career option in a challenging employment environment. Career Development International, 21(3), 214229.doi: 10.1108/CDI-07-2015-0102

Prioste, A., Narciso, I., \& Gonçalves, M. (2012).Questionário de Valores Pessoais Readaptado: Processo de desenvolvimento e contributosiniciais para a validação. Revista Iberoamericana de Diagnóstico y Evaluación - e Avaliaçao Psicológica, 34(1), 175-199.

Sánchez-Teruel, \& Robles-Bello, (2015). Escala de Resiliencia 14 ítems (RS-14): Propiedades Psicométricas de la Versión en Español. Revista Iberoamericana de Diagnóstico y Evaluación - e Avaliaçao Psicológica, 40(2), 103-113.

Savickas, M. L. (2013). The theory and practice of career construction. In S. D. Brown \& R. W. Lent (Eds.), Career development and counselling: Puttingtheory and research to work ( $2^{\text {a }}$ Ed.) (pp. 147-183). Hoboken: Wiley.

Schlossberg, N. K. (2005). Aider les consultants à faire face aux transitions: Le cas particulier des non événements. L'orientation Scolaireet Professionnelle, 34(1), 85-101. 
Shapero, A., \& Sokol, L. (1982). The social dimensions of entrepreneurship. En C. Kent, D. Sexton, \& K. Vesper (Eds.), Encyclopedia of entrepreneurship (pp. 72-90). Englewood Cliffs, NJ: Prentice may.

Sørensen, J. B., \& Sharkey, A. (2014). Entrepreneurship as a mobility process. American SociologicalReview, 79(2), 328349. doi: 10.1177/0003122414521810

Suárez Ortega, M., Cortés Pascual, P. A., \& Sánchez García, M. F. (2015). Orientación para la construcción de la carrera profesional desde perfiles de emprendimiento. En AIDIPE (Ed.), Investigar con y para la sociedad, Vol. 1 (pp. 513-520). Cádiz: Asociación Interuniversitaria de Investigación Pedagógica.

Super, D. E. (1990). A life-span, lifespaceapproach to careerdevelopment. In D. Brown, L. Brooks, \& Associates (orgs.), Careerchoice and development (pp. 197-261). San Francisco: Jossey-Bass.

Taveira, M. C. (2013). Promoción de la empleabilidad profesional en la vida adulta. El papel de los Seminarios de Gestión Personal de la carrera. En P. Figuera Gazo (Ed.), Orientación profesional y transiciones en el mundo global. Innovaciones en orientación sistémica y en gestión personal de la carrera (pp. 161-192). Barcelona: Laertes.

Tolentino, L. R., Garcia, J. M., Lu, V. N., Restubog, S. L. D., Bordia, P., \& Plewa, C. (2014). Career adaptation: The relation of adaptability to goal orientation, proactive personality, and career optimism. Journal of Vocational Behavior, 84 (1), 39-48. doi: 10.1016/j.jvb.2013.11.004

Tomlinson, J., Baird, M., Berg, P., \& Cooper, R. (2016). Conceptualising flexible careers across the life course. Human Relations, 68 (11), 1797-1800. doi: 10.1177/001872671561 2986a

Trejo López, E. (2015). Perfil psicosocial de la persona emprendedora (Tesis doctoral). Valladolid: Universidad de Valladolid.

Valencia Agudelo, G. D. (2012). Autoempleo y emprendimiento. Una hipótesis de trabajo para explicar una de las estrategias adoptadas por los gobiernos para hacer frente al progreso del mercado. Semestre económico, 15(32), 103-128. 